\title{
Componentes de la calidad del servicio en las universidades públicas venezolanas
}

\author{
Components of service quality in venezuelan public universities
}

\begin{tabular}{l|r}
\cline { 2 - 2 } & Antonio Alfonzo \\
\hline $\begin{array}{l}\text { Artículo recibido en septiembre 2020 } \\
\text { Arbitrado en octubre 2020 }\end{array}$ & antonioalfonzo1958@gmail.com \\
$\begin{array}{l}\text { Aceptado en diciembre } 2020 \\
\text { Publicado en enero } 2021\end{array}$ & ORCID: 0000-0002-9010-2873 \\
& $\begin{array}{l}\text { Universidad del Zulia } \\
\text { Maracaibo - Venezuela }\end{array}$
\end{tabular}

RESUMEN $\mid$ El objetivo se enmarco en describir los componentes de calidad del servicio en las universidades públicas venezolanas. Metodológicamente se contextualizo dentro de la investigación descriptiva, con diseño no experimental, de campo y transeccional. Se tomó en cuenta una población intencional constituida por tres (03) universidades públicas, ubicadas en la Costa Oriental del Lago, de la cual se extrajo dos subpoblaciones. Como técnica de recolección de datos se utilizó la encuesta y el censo poblacional, mediante la aplicación de dos instrumentos del tipo cuestionario, los dos fueron validados por expertos y la confiabilidad a través del coeficiente Alfa Cronbach, cuyos resultados fueron de 0,91 y 0,66 respectivamente. El análisis de los datos se realizó mediante la estadística descriptiva, a través de las frecuencias relativas y la media aritmética. Se evidencio que quienes evalúan la calidad lo realizan a través de cinco componentes: tangibilidad, fiabilidad, capacidad de respuesta, seguridad y empatía.

Palabras clave:

Capacidad de respuesta, componentes de calidad del servicio, empatía, fiabilidad, seguridad, tangibilidad

ABSTRACT

Keywords:
The objective was framed in describing the components of service quality in venezuelan public universities. Methodologically it was contextualized within descriptive research, with a non-experimental, field and transectional design. An intentional population made up of three (03) public universities, located on the Eastern Lake Coast, was taken into account, from which two subpopulations were extracted. The survey and population census were used as the data collection technique, by means of the application of two questionnaire-type instruments, both were validated by experts and the reliability through the Alpha Cronbach coefficient, whose results were 0.91 and 0, 66 respectively. Data analysis was performed using descriptive statistics, using relative frequencies and arithmetic mean. It was evident that those who assess quality do so through five components: tangibility, reliability, responsiveness, security and empathy.

Responsiveness, service quality components, empathy, reliability, security, tangibility 


\section{INTRODUCCIÓN}

En un mundo tan globalizado como el actual, es necesario que las organizaciones comprendan el significado de la calidad del servicio y que, además, identifiquen sus ventajas distintivas que las hagan convenientes $y$ diferentes a la competencia. Por tal razón, calidad del servicio constituye una herramienta estratégica que, si es debidamente aplicada, permite visualizar un horizonte más amplio en la búsqueda de la mejora continua, la excelencia y el aumento del nivel competitivo, orientando los esfuerzos hacia la satisfacción de los clientes.

Al respecto Ruiz (2001) considera que la calidad y más concretamente la gestión de la calidad del servicio, es hoy un requisito imprescindible para competir en el ámbito empresarial mundial, ya que las implicaciones que tiene en la cuenta de resultados, tanto en el corto como en el largo plazo, son muy positivas. De esta forma, la calidad del servicio se convierte en un elemento estratégico que confiere una ventaja diferenciadora y perdurable en el tiempo a aquellas empresas que tratan de alcanzarlas.

Desde estas perspectivas, es necesario que las organizaciones analicen y evalúen la calidad del servicio ofrecido, a fin de responder a las necesidades de los clientes, mediante la búsqueda de nuevas estrategias gerenciales orientadas a proveer excelencia en la prestación del servicio.

Las instituciones de educación universitaria venezolanas no escapan a esta realidad, por lo que deben conocer y adecuar sus capacidades en las estrategias utilizadas para la prestación de un servicio con calidad, basadas en cuatro factores: la educación, la experiencia, las habilidades naturales y la actitud; las cuales pueden ser combinadas con la organización, los sistemas, la estructura, la cultura, el ambiente laboral y la investigación (Sotomayor, 2011).

En el caso específico de las universidades públicas de la Costa Oriental del Lago, éstas han evolucionado en los últimos años hacia modelos organizacionales cuya estructura está orientada a fomentar la generación de conocimiento. Como resultado de ello, hoy en día la demanda estudiantil se ha incrementado en estas instituciones, las cuales atienden no solo a los estudiantes que viven en sus adyacencias, sino también a estudiantes foráneos de estados aledaños y hasta de países hermanos.

Consecuentemente, estas instituciones deben preocuparse por diseñar, desarrollar y ejecutar planes para evitar el deterioro progresivo de sus capacidades operativas, siendo una de estas el servicio del transporte estudiantil. En este sentido, Izquierdo y otros (2004), consideran que el servicio del transporte debe contemplarse desde una óptica integral, ya que no es independiente de otras políticas sectoriales: el transporte no es un fin en sí mismo, sino un medio para alcanzar determinados destinos, donde satisfacer un conjunto de necesidades.

Para Cendreros y Truyols (2008), los servicios de transporte se caracterizan como sistemas productivos con una producción no almacenable, es decir, en la mayoría de los casos, adecuar la producción a la demanda. Sin embargo, su dimensión social es muy importante, lo que lleva a encontrarse muy intervenidos por el estado.

En efecto, la experiencia demuestra que el presupuesto ordinario asignado a las universidades públicas imposibilita la adquisición de nuevas unidades autobuseras y que las flotas de estas unidades solo pueden ser ampliadas, a través de las donaciones que 
realiza el Estado por medio de la Oficina de Planificación del Sector Universitario (OPSU).

Tomando en consideración los señalamientos planteados, el investigador a través de la técnica de la observación, además de la revisión documental de algunas estadísticas pudo constatar que las universidades públicas de la Costa Oriental del Lago poseen un alto número de estudiantes que requieren del servicio de transporte, el cual no es cubierto en su totalidad, a pesar que estas han realizado diferentes intentos para conducir sus modelos gerenciales con calidad; sin embargo, aún es escasa la orientación hacia ellos.

Los usuarios cada día son más críticos, exigentes y están dispuestos a luchar por un servicio que consideren satisfagan sus necesidades con la calidad requerida. En el transcurrir del tiempo sus exigencias en cuanto a calidad aumentan, al punto de exigir lo mejor. Al respecto plantea Setó (2004), existen diversos factores o características que hacen variar la calidad del servicio, la cual se evalúa durante todo el proceso de prestación del mismo, dicha evaluación la hace el cliente por comparación entre lo requerido y lo obtenido.

Desde esa perspectiva se realiza, a través de esta investigación una descripción de los componentes de calidad del servicio en las universidades públicas venezolanas, específicamente las ubicadas en la Costa Oriental del Lago en su departamento de transporte.

\section{Calidad del servicio}

La definición de calidad ha resultado ser particularmente compleja en el ámbito de los servicios, esto se debe a la dificultad que se deriva de la naturaleza intangible de los mismos.
Sin embargo, gran cantidad de autores convergen en plantear que la calidad del servicio es la brecha que existe entre las expectativas que se forma el cliente sobre el servicio, antes de adquirirlo, y la percepción de éste después de recibirlo. Esto implica que la calidad del servicio debe estar basada fundamentalmente en las percepciones que los clientes tienen sobre el mismo (Setó, 2004).

De modo que, se puede definir la calidad del servicio como la comparación entre lo que el cliente espera recibir, es decir, sus expectativas, y lo que él realmente recibe o percibe que recibe, dicho de otro modo, el desempeño o percepción del resultado del servicio que recibe (Barroso (2000), citado por Setó, 2004).

En el mismo orden de ideas para Albrecht y Bradford (2004: 84), la calidad de servicio es "Una filosofía, un proceso de pensamiento como un conjunto de valores, actitudes, motivaciones y un conjunto de métodos encaminados al mejoramiento continuo de los productos y los servicios ofrecidos, que redunda en la obtención de la lealtad del cliente y en la consecución de un beneficio económico para la empresa".

En este orden de ideas para Riveros (2007) la calidad del servicio es el proceso de dirección, administración, control y asignación de recursos de las actividades o procesos que se llevan a cabo durante la prestación de un servicio con la finalidad de satisfacer las necesidades $y$ expectativas de los clientes.

En concordancia con lo antes expuesto, para el investigador la calidad del servicio es una de las estrategias más determinantes para lograr diversos beneficios en las instituciones universitarias, como lo son la ventaja competitiva, el incremento de la productividad, la motivación del personal y la lealtad de los 
clientes. Sin embargo, la calidad del servicio no es estática en el tiempo, sino que va evolucionando o deteriorándose, razón por la cual debe ser gestionada, evaluándose y midiéndose constantemente a fin de corregir oportunamente las posibles desviaciones en la prestación del servicio, así como establecer criterios de mejora continua.

\section{Componentes de calidad del servicio}

Según Lamb (2006), la relación empresa cliente es un criterio estandarizado en todos los niveles de una organización para establecer, mantener y profundizar las relaciones con el cliente, quienes evalúan la calidad por medio de cinco componentes: tangibilidad, fiabilidad, capacidad de respuesta, seguridad y empatía.

A su vez, Ruiz (2004) considera que si una organización pretende alcanzar altos niveles en la calidad del servicio que suministra, debe prestar una especial atención a los atributos en los que se fijan los clientes para juzgarla, incluye entre ellos: tangibilidad, fiabilidad, capacidad de respuesta, seguridad y empatía.

En este recorrido de ideas, para el investigador, con la descripción de esta dimensión se pretende obtener información relevante que le indicará en qué aspectos debe centrar los esfuerzos para que sean realmente apreciada la calidad del servicio en el departamento de transporte de las universidades públicas de la Costa Oriental del Lago.

\section{Tangibilidad}

Según Zeithaml y otros (2009), los tangibles se definen como la apariencia de las instalaciones físicas, equipo, personal y materiales de comunicación, además proporcionan representaciones físicas o imágenes del servicio que los clientes, en particular los nuevos, usaran para evaluar la calidad. Las industrias de servicio que enfatizan los tangibles en sus estrategias incluyen servicios en los que el cliente visita el establecimiento para recibir el servicio, como restaurantes y hoteles, tiendas minoristas y compañías de entretenimiento.

En el mismo orden de ideas, para Cottle (2001), la tangibilidad es el aspecto visible del servicio, incluye desde las instalaciones y equipos hasta la apariencia física del personal, puesto que representa la imagen de la calidad. Básicamente se refiere a la evidencia física, los elementos o artefactos que intervienen en el servicio, tales como: folletos, boletines informativos, contratos, mobiliarios, equipos entre otros.

A efecto de la presente investigación, las universidades públicas de la Costa Oriental del Lago al momento de ofrecer sus servicios de una forma directa suministran a los participantes elementos tangibles en su servicio como material de apoyo, facilidad de acceso al uso de tecnologías como computadores y/o equipos de proyección, que formaran parte integral del servicio ofrecido. A pesar de ofrecer servicio de educación que por su naturaleza son intangibles, requiere de elementos tangibles para poder llevar a cabo las actividades a los participantes, en el caso específico de los departamentos de transporte, se hace mención como tangibilidad las condiciones de las unidades de transporte utilizadas.

\section{Fiabilidad}

Para Cottle (2001), la fiabilidad es la habilidad que posee la organización para suministrar el servicio prometido en forma confiable, segura cuidadosa; incluye, además, la 
rapidez. De igual manera, Zeithaml y otros (2009) consideran la fiabilidad el determinante más importante de las percepciones de la calidad de servicio, definiéndose como la capacidad para ejecutar el servicio prometido en forma segura y precisa. En un sentido más amplio, confiabilidad significa que la compañía cumple sus promesas, promesas sobre entrega, suministro del servicio, solución de problemas fijación de precios.

Así las cosas, para el investigador la fiabilidad estará referida a la capacidad que tienen las universidades públicas de la Costa Oriental del Lago, para desarrollar el servicio que se promete de forma digna, segura y precisa. Al desarrollar planes de formación por su parte, estas hacen esfuerzos para demostrar a sus participantes que sus productos y servicios ofrecidos garantizan un porcentaje o grado de confianza sobre los resultados futuros del conocimiento recibido.

\section{Capacidad de respuesta}

Según Cottle (2001), la capacidad de respuesta se refiere a la calidad que muestra el proveedor de servicio para ayudar a sus clientes y suministrar un servicio rápido. Incluye el cumplimiento a tiempo de los compromisos contraídos y la accesibilidad concerniente a la posibilidad y facilidad de entrar en contacto con el cliente.

En este orden de ideas, Zeithaml y otros (2009) consideran la capacidad de respuesta la disposición de ayudar a los clientes y a proporcionar un servicio expedito, este componente hace hincapié en la atención y prontitud al tratar las solicitudes, preguntas, quejas y problemas del cliente. Se evidencia por la cantidad de tiempo que tienen que esperar por la asistencia, las respuestas a las preguntas o la atención de problemas.

Para las universidades públicas de la Costa Oriental del Lago la capacidad de respuestas, estará dada por el trato hacia el usuario y en la toma de decisiones del departamento de transporte ante las eventualidades suscitadas en el servicio que ofrece.

\section{Seguridad}

Para Zeithaml y otros (2009), la seguridad se define como el conocimiento y cortesía de los empleados, así como la capacidad que tiene la empresa y sus empleados para inspirar al cliente credibilidad además de confianza. Es probable que este componente sea importante en particular para los servicios que los clientes perciben como de alto riesgo o para servicios de los cuales se sienten inseguros sobre su capacidad para evaluar los resultados; por ejemplo, servicios de banca, seguros, corretaje, médicos y legales.

De la misma manera, Cottle (2001) la define como el sentimiento que tienen los clientes, en el cual los problemas están en buenas manos y serán resueltos de la mejor manera posible. Incluye la confianza, credibilidad, integridad y honestidad, con el objeto de demostrarle al cliente que estén protegiendo sus intereses.

El investigador, considera la seguridad como un valor intangible por excelencia, donde el participante puede sentir que su participación en los departamentos de transporte de las universidades públicas de la Costa Oriental del Lago, garantizan seguridad. Aspectos tales como: puntualidad, espacio físico, ambiente puede influir fuertemente en ese sentimiento de seguridad que, para el usuario puede objetar; por ejemplo; considerar el transporte seguro por 
su condición, pero si el usuario observa daño físico o filtraciones, sin aplicar estudios profundos o conocimiento científico en materia de mantenimiento, con solo observar esos detalles sentirá que no es seguro trasladarse en ese tipo de transporte por lo que descalificará o no a la institución.

\section{Empatía}

Referida a la información requerida, ofreciéndoles a los clientes cuidados, disposición y atención personalizada, involucra un fuerte compromiso e implicación conociendo y comprometiendo a fondo sus características, necesidades personales y requerimientos, así como también implica la cortesía, la consideración, el respeto, la comunicación y el tiempo de los clientes, hasta que se establezca una atmósfera de amistad en el contacto personal. Por medio de una buena comunicación se puede mejorar la empatía, manteniendo a los clientes informados, utilizando un lenguaje que pueden entender $y$ escuchándolos (Cottle, 2001).

Zeithaml y otros (2009) la definen como la atención individualizada cuidadosa que la empresa proporciona a cada uno de sus clientes. La esencia de la empatía es trasmitir, por medio de un servicio personalizado o adaptado al gusto del cliente, que los clientes son únicos y especiales y que se entienden sus necesidades, ya que cada uno de los clientes tienen la necesidad de sentirse comprendidos e importantes para la empresa que les proporcionan el servicio.

A efecto de esta investigación, la empatía es la capacidad de las universidades públicas de la Costa Oriental del Lago, en tratar a los clientes como individuos y colocarse en su lugar. Las personas que laboran dentro de los departamentos de transporte, deben demostrar que son capaces de escuchar a sus participantes para entender sus inquietudes, problemas y motivaciones, lo cual hace que se incorpore un valor agregado a la calidad del servicio, se genera reconocimiento de sus éxitos y corrige a tiempo sus fallas a la hora de satisfacer esas necesidades.

El participante puede manifestar si son capaces de reconocer sus fortalezas $y$ debilidades y eso genera popularidad positiva o negativa sobre la misma. Por otra parte, la institución debe anticiparse a las necesidades de sus participantes y para ello debe crear empatía con ellos para interactuar y mejorar sus procesos garantizando estabilidad y éxito en su desarrollo institucional.

\section{MÉTODO}

Metodológicamente se contextualizo dentro de un tipo de investigación analítica, con diseño no experimental, de campo y transeccional. Para efectos del estudio, se tomó en cuenta una población intencional constituida por tres (03) universidades públicas, ubicadas en la Costa Oriental del Lago: Universidad del Zulia, núcleo Costa Oriental del Lago, Universidad Nacional Experimental "Rafael María Baralt", y la Universidad Politécnica Territorial del Zulia, sede Cabimas.

De ellas, se extrajo dos sub-poblaciones, la $A$, referida a los usuarios de los servicios de transporte y la sub-población $B$, referida a los coordinadores y jefes de transporte, de dichas instituciones. Esta última constituida por ocho sujetos, por lo que se consideró en su totalidad, mientras que para la sub-población A se utilizó un muestreo aleatorio por conglomerados, resultando una muestra constituida por 675 sujetos. 
Como técnica de recolección de datos se utilizó la encuesta para la sub-población $\mathrm{A}$ y el censo poblacional para la $B$, mediante la aplicación de dos instrumentos del tipo cuestionario estructurado, ambos contentivos de por 10 Ítemes, los dos fueron validados por cinco expertos y para la confiabilidad de los mismos se aplicó a través del coeficiente Alfa Cronbach, cuyos resultados fueron de 0,91 para la sub-población A y 0,66 para la sub-población
B ubicándose como muy alta y alta confiabilidad respectivamente.

El análisis de los datos se realizó mediante la estadística descriptiva, a través de las frecuencias relativas y la media aritmética. En cuanto a la interpretación de los valores obtenidos en los indicadores y dimensión, en el cuadro 1 se presenta el baremo para identificar el nivel de respuesta según el rango de apreciación establecido para cada intervalo.

Cuadro 1. Categoría de análisis para la interpretación del promedio

\begin{tabular}{ccc}
\hline Dimensión & Rango para la media & Nivel de respuesta para la dimensión y sus indicadores \\
\hline & $4.20-5.00$ & Muy alta presencia \\
Componentes & $3.40-4.20$ & Alta presencia \\
& $2.60-3.40$ & Moderada presencia \\
& $1.80-2.60$ & Baja presencia \\
& $1.00-1.80$ & Muy baja presencia \\
\hline
\end{tabular}

Fuente: Elaboración propia (2019)

De igual manera, para el análisis de las frecuencias el investigador consideró conveniente fijar un baremo de interpretación, el cual se recoge en el cuadro 2 .

Cuadro 2. Tendencia de análisis para la interpretación de la frecuencia relativa

\begin{tabular}{cc}
\hline Alternativa & Tendencia \\
\hline TD $+\mathrm{DA} \geq 70 \%$ & La tendencia se considera positiva \\
$45 \% \leq \mathrm{TD}+\mathrm{DA}<70 \%$ & La tendencia se considera neutral \\
$\mathrm{TD}+\mathrm{DA}<45 \%$ & La tendencia se considera negativa \\
\hline
\end{tabular}

Fuente: Elaboración propia (2019)

\section{RESULTADOS}

Con respecto al objetivo, orientado a describir los componentes de calidad del servicio en las universidades públicas venezolanas, específicamente en la Costa Oriental del Lago en el departamento de transporte, los resultados obtenidos se presentan en la tabla 1 e indican según las respuestas proporcionadas por las poblaciones encuestadas, en relación al indicador tangibilidad, que existe una moderada presencia según opinión de los estudiantes con promedio de 2,95, mientras que los coordinadores/jefes de transporte su promedio de 3,50 ubicándola en una categoría de alta presencia. 
Tabla 1. Indicador: Tangibilidad

\section{Estudiantes}

Ítems de cuestionario

El transporte se encuentra en buen estado

El personal de transporte proyecta buena imagen
El transporte se encuentra en buen estado

Usted proyecta buena imagen

\section{Ítems del indicador \\ Estudiantes}

\begin{tabular}{|c|c|c|c|c|}
\hline \multirow{3}{*}{ Alternativas } & & & \multicolumn{2}{|c|}{ transporte } \\
\hline & 1 & 2 & 1 & 2 \\
\hline & Fr & Fr & Fr & $\mathbf{F r}$ \\
\hline TD & 7 & 18 & 0 & 100 \\
\hline MD & 21 & 24 & 0 & 0 \\
\hline NDNED & 21 & 35 & 0 & 0 \\
\hline MED & 26 & 14 & 100 & 0 \\
\hline TED & 25 & 9 & 0 & 0 \\
\hline TOTAL & 100 & 100 & 100 & 100 \\
\hline \multirow[t]{2}{*}{ ALTERNATIVA } & 28 & 42 & 0 & 100 \\
\hline & $\mathrm{TD}+\mathrm{DA}<45 \%$ & $\mathrm{TD}+\mathrm{DA}<45 \%$ & $\mathrm{TD}+\mathrm{DA}<45 \%$ & $\mathrm{TD}+\mathrm{DA} \geq 70 \%$ \\
\hline TENDENCIA & Negativa & Negativa & Negativa & Positiva \\
\hline PROMEDIO/ÍTEMES & 2,6 & 3,3 & 2 & 5 \\
\hline CATEGORÍA & $\begin{array}{l}\text { Moderada } \\
\text { presencia }\end{array}$ & $\begin{array}{l}\text { Moderada } \\
\text { presencia }\end{array}$ & Baja presencia & $\begin{array}{l}\text { Muy alta } \\
\text { presencia }\end{array}$ \\
\hline PROMEDIO/INDICADOR & Modera & 95 & $\begin{array}{r}3 \\
3\end{array}$ & 0 \\
\hline
\end{tabular}

Fuente: Elaboración propia (2019)

De acuerdo a los valores obtenidos, se observa baja concentración de respuestas en las opciones totalmente de acuerdo y de acuerdo, denotado por la suma de las frecuencias relativas cuyos valores solo ascienden a $28 \%$ en el ítem 1 para los estudiantes $y, 0 \%$ para el de los coordinadores/jefes de transporte, marcando tendencia negativa en cuanto a si el transporte se encuentra en buen estado. Asimismo, al referirse si el personal de transporte proyecta buena imagen, las opciones totalmente de acuerdo y de acuerdo, denotado por la suma de las frecuencias relativas solo se concentró en un $42 \%$ marcando tendencia negativa de las respuestas emitidas por los estudiantes, no obstante, los coordinadores/jefes de transporte opinaron en un $100 \%$ estar totalmente de acuerdo y de acuerdo que ellos proyectan buena imagen marcando tendencia positiva de las respuestas.

Con base a lo descrito, puede decirse entonces que, las universidades públicas de la Costa Oriental del Lago al momento de ofrecer sus servicios de una forma directa buscan suministrar a la comunidad universitaria elementos tangibles en su servicio, que formaran parte integral del mismo y que en el caso específico de los departamentos de transporte, se hace mención las condiciones de las unidades de transporte utilizadas, así entonces, en opinión de ambos sectores en términos generales la tangibilidad, no se percibe totalmente como un criterio esencial para la calidad de las instituciones bajo estudio, siendo este un aspecto visible del servicio, que incluye desde las 
instalaciones y equipos hasta la apariencia física del personal, puesto que representa la imagen de la calidad, tal como lo expresa Cottle (2001).

Seguidamente, en la tabla 2 , se resume el comportamiento para el indicador fiabilidad, donde se observa que alcanzó un promedio de 2,80 y 2,00 según opinión de los estudiantes y Coordinadores/jefes de transporte respectivamente, indicando moderada y baja presencia, acompañada de una tendencia negativa de las respuestas obtenidas, implicando esto a juicio del investigador que no se logra brindar el servicio que se promete de forma digna, segura y precisa.
De manera específica, puede observarse que solo el $27 \%$ de los estudiantes están totalmente de acuerdo y de acuerdo de que la institución le ofrece todas las rutas que se requiere para su traslado, no obstante, los Coordinadores/jefes de transporte no alcanzaron \%, para ambas poblaciones la tendencia se ubicó en negativa. Por otra parte, al referirse si la institución le brinda información clara en el momento oportuno, los estudiantes opinan estar totalmente de acuerdo y de acuerdo en un $21 \%$, mientras que los coordinadores/jefes de transporte no obtuvieron \%, indicando tendencia negativa de las respuestas obtenidas.

Tabla 2. Indicador: Fiabilidad

\section{Ítems de cuestionario}

Estudiantes

\section{Coordinadores/jefes de transporte}

La institución le ofrece todas las rutas que se requiere para su traslado

La institución ofrece todas las rutas que se requiere para el traslado del usuario

La institución le brinda información clara en el momento oportuno

La institución brinda información clara en el momento oportuno

\begin{tabular}{|c|c|c|c|c|}
\hline \multirow{3}{*}{ Alternativas } & \multicolumn{2}{|c|}{ Estudiantes } & \multicolumn{2}{|c|}{$\begin{array}{c}\text { Coordinadores/jefes de } \\
\text { transporte }\end{array}$} \\
\hline & 3 & 4 & 3 & 4 \\
\hline & $\mathbf{F r}$ & $\mathbf{F r}$ & Fr & $\mathbf{F r}$ \\
\hline TD & 7 & 17 & 0 & 0 \\
\hline MD & 20 & 24 & 0 & 0 \\
\hline NDNED & 20 & 30 & 0 & 100 \\
\hline MED & 14 & 17 & 0 & 0 \\
\hline TED & 39 & 12 & 100 & 0 \\
\hline TOTAL & 100 & 100 & 100 & 100 \\
\hline \multirow[t]{2}{*}{ ALTERNATIVA } & 27 & 21 & 0 & 0 \\
\hline & $\mathrm{TD}+\mathrm{DA}<45 \%$ & $\mathrm{TD}+\mathrm{DA}<45 \%$ & $\mathrm{TD}+\mathrm{DA}<45 \%$ & $\mathrm{TD}+\mathrm{DA}<45 \%$ \\
\hline TENDENCIA & Negativa & Negativa & Negativa & Negativa \\
\hline PROMEDIO/ÍTEMES & 2,4 & 3,2 & 1 & 3 \\
\hline CATEGORÍA & $\begin{array}{l}\text { Moderada } \\
\text { presencia }\end{array}$ & $\begin{array}{l}\text { Moderada } \\
\text { presencia }\end{array}$ & $\begin{array}{l}\text { No hay } \\
\text { presencia }\end{array}$ & $\begin{array}{l}\text { Moderada } \\
\text { presencia }\end{array}$ \\
\hline PROMEDIO/INDICADOR & \multirow{2}{*}{\multicolumn{2}{|c|}{$\begin{array}{c}2,80 \\
\text { Moderada presencia }\end{array}$}} & \multicolumn{2}{|c|}{2} \\
\hline CATEGORÍA & & & Baja p & esencia \\
\hline
\end{tabular}

Fuente: Elaboración propia (2019) 
Al contextualizar los datos obtenidos con las teorías de Cottle (2001), para quien la fiabilidad es la habilidad que posee la organización para suministrar el servicio prometido en forma confiable, segura cuidadosa; incluye, además, la rapidez, es evidente que, las instituciones universitarias bajo estudio, deben hacer esfuerzos para garantizar un porcentaje o grado de confianza sobre el servicio prestado.

En lo concerniente al indicador capacidad de respuesta, se observa, en la tabla 3, un promedio de 3,00 en opinión de los estudiantes y 3,50 según los coordinadores/jefes de transporte, ubicándola en la categoría moderada presencia y alta presencia; como dimensión de la calidad del servicio en las instituciones analizadas.

Al detalle se observa que, respecto a si el personal de transporte lo atiende oportunamente, la suma de las opciones positivas, totalmente de acuerdo y de acuerdo, en opinión de los estudiantes se sitúa en la tendencia negativa 32\%, igual tendencia al observar la opinión de los coordinadores/jefes de transporte quienes no alcanzaron \% en estas opciones al referir si atiende oportunamente a los usuarios, cuando este lo requiere.

Tabla 3. Indicador: Capacidad de respuesta

\begin{tabular}{|c|c|c|c|c|}
\hline \multicolumn{5}{|c|}{ Ítems de cuestionario } \\
\hline \multicolumn{2}{|c|}{ Estudiantes } & \multicolumn{3}{|c|}{ Coordinadores/jefes de transporte } \\
\hline \multicolumn{2}{|c|}{$\begin{array}{l}\text { El personal de transporte lo } \\
\text { oportunamente }\end{array}$} & \multicolumn{3}{|c|}{$\begin{array}{l}\text { Atiende oportunamente a los usuarios, cuando este } \\
\text { lo requiere }\end{array}$} \\
\hline \multicolumn{2}{|c|}{$\begin{array}{l}\text { El personal de transporte aclara satisfactoriamente } \\
\text { sus dudas referentes a los servicios ofrecidos }\end{array}$} & \multicolumn{3}{|c|}{$\begin{array}{l}\text { Usted aclara satisfactoriamente a los usuarios, las } \\
\text { dudas referentes a los servicios ofrecidos }\end{array}$} \\
\hline \multirow{3}{*}{ Alternativas } & \multicolumn{2}{|c|}{$\begin{array}{l}\text { Ítems del indicador } \\
\text { Estudiantes }\end{array}$} & \multicolumn{2}{|c|}{$\begin{array}{l}\text { Coordinadores/jefes de } \\
\text { transporte }\end{array}$} \\
\hline & 5 & 6 & 5 & 6 \\
\hline & $\mathbf{F r}$ & Fr & $\mathbf{F r}$ & $\mathbf{F r}$ \\
\hline TD & 12 & 12 & 0 & 0 \\
\hline MD & 20 & 18 & 0 & 100 \\
\hline NDNED & 36 & 41 & 100 & 0 \\
\hline MED & 20 & 19 & 0 & 0 \\
\hline TED & 12 & 10 & 0 & 0 \\
\hline TOTAL & 100 & 100 & 100 & 100 \\
\hline \multirow[t]{2}{*}{ ALTERNATIVA } & 32 & 30 & 0 & 100 \\
\hline & $\mathrm{TD}+\mathrm{DA}<45 \%$ & $\mathrm{TD}+\mathrm{DA}<45 \%$ & $\mathrm{TD}+\mathrm{DA}<45 \%$ & $T D+D A \geq 70 \%$ \\
\hline TENDENCIA & Negativa & Negativa & Negativa & Positiva \\
\hline PROMEDIO/ÍTEMES & 3 & 3 & 3 & 4 \\
\hline CATEGORÍA & $\begin{array}{l}\text { Moderada } \\
\text { presencia }\end{array}$ & $\begin{array}{l}\text { Moderada } \\
\text { presencia }\end{array}$ & $\begin{array}{l}\text { Moderada } \\
\text { presencia }\end{array}$ & Alta presencia \\
\hline PROMEDIO/INDICADOR & $\mathbf{3}$ & & & \\
\hline CATEGORÍA & Moderada & presencia & Alta $\mathbf{p}$ & sencia \\
\hline
\end{tabular}

Fuente: Elaboración propia (2019) 
Asimismo, se observa que solo el $30 \%$ de los estudiantes están totalmente de acuerdo y de acuerdo que el personal de transporte aclara satisfactoriamente sus dudas referentes a los servicios ofrecidos, marcando una tendencia negativa de las respuestas, contraria al de los coordinadores/jefes de transporte, cuya tendencia es positiva, al considerar estos en un $100 \%$ estar totalmente de acuerdo y de acuerdo que ellos aclaran satisfactoriamente a los usuarios, las dudas referentes a los servicios ofrecidos.

Con base a lo descrito, puede decirse entonces que la capacidad de respuestas de las universidades públicas venezolanas de la Costa Oriental del Lago, no siempre está dada por el trato hacia el usuario y en la toma de decisiones que el departamento de transporte debe hacer según sean las eventualidades suscitadas en el servicio que ofrece, para lograr con ello proporcionar un servicio expedito, en la atención y prontitud al tratar las solicitudes, preguntas, quejas y problemas del cliente, como es reseñado por Zeithaml y otros (2009).

Continuando con la exposición de los resultados, se presenta en la tabla 4 los correspondientes al indicador seguridad, evidenciándose un promedio de 3,60 en opinión de los estudiantes y 4,50 según los coordinadores/jefes de transporte, ubicándola en la categoría alta presencia y muy alta presencia respectivamente.

Tabla 4. Indicador: Seguridad

\section{Estudiantes}

La institución cuenta con personal capacitado para brindar los servicios requeridos

El conductor de la unidad de transporte inspira confianza

\begin{tabular}{|c|c|c|c|c|}
\hline \multirow{3}{*}{ Alternativas } & \multicolumn{2}{|c|}{ Estudiantes } & \multicolumn{2}{|c|}{$\begin{array}{c}\text { Coordinadores/jefes de } \\
\text { transporte }\end{array}$} \\
\hline & 7 & 8 & 7 & 8 \\
\hline & Fr & $\mathbf{F r}$ & $\mathbf{F r}$ & $\mathbf{F r}$ \\
\hline TD & 22 & 27 & 0 & 100 \\
\hline MD & 35 & 28 & 100 & 0 \\
\hline NDNED & 26 & 33 & 0 & 0 \\
\hline MED & 12 & 5 & 0 & 0 \\
\hline TED & 5 & 7 & 0 & 0 \\
\hline TOTAL & 100 & 100 & 100 & 100 \\
\hline ALTERNATIVA & $\begin{array}{c}57 \\
45 \% \leq \mathrm{TD}+\mathrm{DA}< \\
70 \%\end{array}$ & $\begin{array}{c}55 \\
45 \% \leq \mathrm{TD}+\mathrm{DA}< \\
70 \%\end{array}$ & $\begin{array}{c}100 \\
T D+D A \geq 70 \%\end{array}$ & $\begin{array}{c}100 \\
T D+D A \geq 70 \%\end{array}$ \\
\hline TENDENCIA & Neutral & Neutral & Positiva & Positiva \\
\hline PROMEDIO/ÍTEMES & 3,6 & 3,6 & 4 & 5 \\
\hline CATEGORÍA & Alta presencia & Alta presencia & Alta presencia & $\begin{array}{l}\text { Muy alta } \\
\text { presencia }\end{array}$ \\
\hline PROMEDIO/INDICADOR & & & & \\
\hline CATEGORÍA & Alta $\mathbf{p}$ & sencia & Muy alt & resencia \\
\hline
\end{tabular}

Fuente: Elaboración propia (2019) 
Del mismo modo, se puede observar cómo un $57 \%$ de los estudiantes encuestados señalaron estar totalmente de acuerdo y de acuerdo que la institución cuenta con personal capacitado para brindar los servicios requeridos, denotando una tendencia neutral, mientras que la tendencia para los coordinadores/jefes de transporte se ubica en positiva al referir estos el $100 \%$ en la suma de las respuestas emitidas.

Al mismo tiempo, se muestran una tendencia neutral, al considerar los estudiantes estar totalmente de acuerdo y de acuerdo que el conductor de la unidad de transporte inspira confianza (55\%), aspecto que al ser comparado con la opinión de los coordinadores/jefes de transporte, se ubicó en tendencia positiva al considerar ellos en un 100\% estar totalmente de acuerdo y de acuerdo que inspiran confianza.

Estos resultados son altamente coincidentes con lo manejado por Cottle (2001), quien refiere la seguridad como el sentimiento que tienen los clientes, en el cual los problemas están en buenas manos y serán resueltos de la mejor manera posible. Así las cosas, al contextualizar los resultados con esta teoría, el investigador la visualiza como un valor intangible por excelencia, donde se puede sentir que la participación de quienes hacen vida en los departamentos de transporte de las universidades públicas venezolanas de la Costa Oriental del Lago, garantiza seguridad.
En el orden de presentación de los resultados, se tienen los del indicador empatía. En este sentido, en la tabla 5, se observa un promedio de 3,45 y 3,50 según la posición de los estudiantes y coordinadores/jefes de transporte, ubicándolo en una categoría de alta presencia para ambas poblaciones.

Al desglosar resultados por ítems, se evidencia como el $56 \%$ de los estudiantes encuestados opinaron estar totalmente de acuerdo y de acuerdo que el personal de transporte emplea un lenguaje apropiado al comunicarse con los usuarios del servicio, ubicándolo en una tendencia neutral, mientras que la tendencia de las respuestas emitidas por los coordinadores/jefes de transporten se ubica en positiva, al considerar estos en un $100 \%$ estar totalmente de acuerdo y de acuerdo que utilizan un lenguaje apropiado al comunicarse con los usuarios del servicio.

De igual manera, los estudiantes consideran estar totalmente de acuerdo y de acuerdo en un $45 \%$ que el personal de transporte se esmera por atenderlo adecuadamente, ubicándolo en una tendencia neutral; caso contrario en opinión de los coordinadores/jefes de transporte, cuyas sumas de las alternativas positivas de totalmente de acuerdo y de acuerdo $0 \%$, lo ubican en una tendencia negativa al considerar ellos que no se esmeran por atender adecuadamente a los usuarios del transporte. 
Tabla 5. Indicador: Empatía

\section{Estudiantes \\ Ítems de cuestionario \\ Coordinadores/jefes de transporte}

El personal de transporte emplea un lenguaje Utiliza un lenguaje apropiado al comunicarse con apropiado al comunicarse con los usuarios del los usuarios del servicio servicio

El personal de transporte se esmera por atenderlo Usted se esmera por atender adecuadamente a los adecuadamente usuarios del transporte

\begin{tabular}{|c|c|c|c|c|}
\hline \multirow{3}{*}{ Alternativas } & \multicolumn{2}{|c|}{$\begin{array}{l}\text { Ítems del indicador } \\
\text { Estudiantes }\end{array}$} & \multicolumn{2}{|c|}{$\begin{array}{c}\text { Coordinadores/jefes de } \\
\text { transporte }\end{array}$} \\
\hline & 9 & 10 & 9 & 10 \\
\hline & $\mathbf{F r}$ & $\mathbf{F r}$ & $\mathbf{F r}$ & Fr \\
\hline TD & 28 & 12 & 0 & 0 \\
\hline MD & 28 & 33 & 100 & 0 \\
\hline NDNED & 31 & 32 & 0 & 100 \\
\hline MED & 7 & 11 & 0 & 0 \\
\hline TED & 6 & 12 & 0 & 0 \\
\hline TOTAL & 100 & 100 & 100 & 100 \\
\hline \multirow{2}{*}{ ALTERNATIVA } & 56 & 45 & 100 & 0 \\
\hline & $\begin{array}{c}45 \% \leq \mathrm{TD}+\mathrm{DA}< \\
70 \%\end{array}$ & $\begin{array}{c}45 \% \leq \mathrm{TD}+\mathrm{DA}< \\
70 \%\end{array}$ & $\mathrm{TD}+\mathrm{DA} \geq 70 \%$ & $\mathrm{TD}+\mathrm{DA}<45 \%$ \\
\hline TENDENCIA & Neutral & Neutral & Positiva & Negativa \\
\hline PROMEDIO/ÍTEMES & 3,7 & 3,2 & 4 & 3 \\
\hline CATEGORÍA & Alta presencia & $\begin{array}{c}\text { Moderada } \\
\text { presencia }\end{array}$ & Alta presencia & $\begin{array}{c}\text { Moderada } \\
\text { presencia }\end{array}$ \\
\hline $\begin{array}{l}\text { PROMEDIO/INDICADOR } \\
\text { CATEGORÍA }\end{array}$ & $\begin{array}{r}3 \\
\text { Alta }\end{array}$ & 5 & $\begin{array}{r}3 \\
\text { Alta }\end{array}$ & 50 \\
\hline
\end{tabular}

Fuente: Elaboración propia (2019)

Se evidencia de estos hallazgos que las personas que laboran dentro de los departamentos de transporte, deben demostrar que son capaces de escuchar a sus participantes para entender sus inquietudes, problemas $y$ motivaciones, lo cual hace que se incorpore un valor agregado a la calidad del servicio, se genera reconocimiento de sus éxitos y corrige a tiempo sus fallas a la hora de satisfacer esas necesidades. Logrando con ello trasmitir, por medio de un servicio personalizado del cliente, que se entienden sus necesidades, tal como es expresado por Zeithaml y otros (2009).

Una vez analizados cada uno de los indicadores considerados en la dimensión componentes de calidad del servicio, se pasa a verificar los resultados arrojados en su totalidad. Así entonces, en la tabla 6 se observa un promedio de 3,16 para los estudiantes, con categoría de moderada presencia y de alta presencia con un promedio de 3,40 en opinión de los coordinadores/jefes de transporte. Evidenciándose, la existencia de ciertos indicadores involucrados en el proceso de prestación de servicio, que les permite a los usuarios del transporte medir la calidad del servicio prestado, y a su vez a las universidades bajo estudio conocer las posibles causas que le entorpecen brindar un servicio de completa satisfacción. 
Tabla 6. Dimensión: Componentes de calidad del servicio

\begin{tabular}{lcccc}
\hline \multicolumn{1}{c}{ Indicadores } & \multicolumn{2}{c}{ Estudiantes } & \multicolumn{2}{c}{ Coordinadores/jefes de transporte } \\
& Promedio & Categoría & Promedio & Categoría \\
\hline Tangibilidad & 2,95 & Moderada presencia & 3,50 & Alta presencia \\
Fiabilidad & 2,80 & Moderada presencia & 2,00 & Baja presencia \\
Capacidad de respuesta & 3,00 & Moderada presencia & 3,50 & Alta presencia \\
Seguridad & 3,60 & Alta presencia & 4,50 & Muy alta presencia \\
Empatía & 3,45 & Alta presencia & 3,50 & Alta presencia \\
Promedio & $\mathbf{3 , 1 6}$ & Moderada & $\mathbf{3 , 4 0}$ & Alta presencia \\
\hline
\end{tabular}

Fuente: Elaboración propia (2019)

Los resultados descritos, evidenciaron la presencia de ciertos criterios estandarizados en los niveles de las instituciones bajo estudio para establecer, mantener y profundizar las relaciones con el cliente, quienes evalúan la calidad por medio de cinco componentes: tangibilidad, fiabilidad, capacidad de respuesta, seguridad y empatía, como es señalado por Lamb (2006).

Estos resultados, denotan como en las universidades públicas, las percepciones del cliente son valoraciones subjetivas reales del servicio, y esto depende de la forma como el cliente evalúa el servicio de acuerdo a las dimensiones del mismo, afirmación que se valida al comparar estos resultados con los obtenidos por Gudiño (2014), quien en su investigación sobre la Calidad del servicio en los hospitales tipo IV adscritos a la Secretaría de Salud del estado Zulia, concluyo que existían deficiencias en las dimensiones de la calidad de servicio brindadas en estas instituciones, específicamente en cuanto a la responsabilidad, empatía y tangibles.

Generando de esta manera la insatisfacción de los mismos, por lo que se recomendó implementar estrategias de mejora que incidieran en la calidad de servicio y, por ende, en el bienestar pleno de los mismos. Aspecto que a juicio del investigador también deben ser considerados al momento de realizar las recomendaciones respectivas de la presente investigación, por la moderada presencia que ostentan las respuestas emitidas por los estudiantes y de baja presencia en algunos casos a juicio de los coordinadores/jefes de transporte.

\section{CONCLUSIONES}

En relación al objetivo, el cual fue describir los componentes de calidad del servicio en las universidades públicas venezolanas, se puede concluir que los resultados obtenidos mostraron la presencia de ciertos criterios estandarizados en los niveles de las instituciones bajo estudio para establecer, mantener y profundizar las relaciones con el cliente, quienes evalúan la calidad por medio de cinco componentes: tangibilidad, fiabilidad, capacidad de respuesta, seguridad y empatía.

Respecto a la tangibilidad, se determinó una moderada y alta presencia de los elementos tangibles en su servicio, que forman parte integral del mismo y que en el caso específico de los departamentos de transporte, se hace mención a las condiciones de las unidades de transporte utilizadas. 
Con relación a la fiabilidad, los resultados indican moderada y baja presencia, para brindar el servicio que se promete de forma digna, segura y precisa. Por otra parte, al considerar la capacidad de respuesta, esta evidencio una moderada y alta presencia en la atención y prontitud al tratar las solicitudes, preguntas, quejas y problemas del cliente.

Sobre la seguridad, se registró una alta y muy alta presencia de la participación de quienes hacen vida en los departamentos de transporte de las universidades públicas venezolanas, de la Costa Oriental del Lago, para garantizar la seguridad. Asimismo, se visualizó alta presencia de la empatía de los trabajadores dentro de los departamentos de transporte, quienes son capaces de escuchar a sus participantes para entender sus inquietudes, problemas $y$ motivaciones, lo cual hace que se incorpore un valor agregado a la calidad del servicio.

\section{REFERENCIAS}

Albrecht, K. y Bradford L. (2004), La excelencia del servicio, 3R Editores, México

Barroso, C. (2000). Marketing Relacional. Ed. ESIC, Madrid

Cendrero, B. y Truyols, S. (2008). El Transporte. Aspectos y Tipología. Publicaciones Delta, Madrid

Cottle, P. (2001). El Servicio Centrado en el Cliente. Librería Dos Santos. BarcelonaEspaña
Gudiño, J. (2014). Calidad del servicio en los hospitales tipo IV adscritos a la Secretaría de Salud del estado Zulia. Trabajo de Grado. Maestría en Gerencia de Empresas, Mención Gerencia de Operaciones. Universidad del Zulia. Cabimas. Venezuela

Izquierdo, R., Bartolome, M. y Ramos, R. (2011). Introducción al transporte. Editorial: ets de ingenieros de caminos. España

Lamb, O. (2006). Gestion de la calidad total. Un texto introductorio. Editorial Prentice Hall. Madrid España

Riveros, P. (2007). Sistema de gestión de la calidad del servicio. Sea líder en mercados altamente competitivos. 3ra Edición. ECOE Ediciones. Colombia.

Ruiz, J. (2004). La gestión por calidad total en la empresa. Ediciones Alfa-Omega, Grupo Editor. México

Ruiz, J. (2004). La gestión por calidad total en la empresa. Ediciones Alfa-Omega, Grupo Editor. México

Setó, D. (2004). De la calidad de servicio a la fidelidad del cliente. ESIC Editorial. España

Sotomayor, S (2011). La relevancia valorativa de los intangibles y los valores tecnológicos europeos. Editor Universidad de Cádiz. Servicio de Publicaciones. Servicio Publicaciones UCA

Zeithaml, V., Bitner, M. y Gremler, D. (2009). Marketing de los servicios. Quinta edición. Editorial Mc Graw - Hill. Interamericana. México 